PROCEEDINGS OF THE

AMERICAN MATHEMATICAL SOCIETY

Volume 134, Number 10, October 2006, Pages 2857-2860

S $0002-9939(06) 08518-2$

Article electronically published on May 1, 2006

\title{
ON THE MANIN-MUMFORD CONJECTURE FOR ABELIAN VARIETIES WITH A PRIME OF SUPERSINGULAR REDUCTION
}

\author{
TETSUSHI ITO
}

(Communicated by Michael Stillman)

\begin{abstract}
We give a short proof of the "prime-to- $p$ version" of the ManinMumford conjecture for an abelian variety over a number field, when it has supersingular reduction at a prime dividing $p$, by combining the methods of Bogomolov, Hrushovski, and Pink-Roessler. Our proof here is quite simple and short, and neither $p$-adic Hodge theory nor model theory is used. The observation is that a power of a lift of the Frobenius element at a supersingular prime acts on the prime-to- $p$ torsion points via nontrivial homothety.
\end{abstract}

Let $A$ be an abelian variety over a number field $K$. For an integer $n \geq 1$, let $[n]: A \rightarrow A$ be the multiplication-by- $n$ isogeny on $A$, and $A[n]$ the kernel of $[n]$ acting on the set of $\bar{K}$-valued points $A(\bar{K})$ of $A$, where $\bar{K}$ denotes an algebraic closure of $K$. Let $\operatorname{Tor}(A):=\bigcup_{n \geq 1} A[n]$ be the group of torsion points on $A$. For a prime number $p$, let $\operatorname{Tor}_{p}(A):=\bigcup_{n \geq 1} A\left[p^{n}\right]\left(\operatorname{resp} . \operatorname{Tor}^{p}(A):=\bigcup_{(n, p)=1} A[n]\right)$ denote the subgroup of $\operatorname{Tor}(A)$ consisting of elements which have $p$-power order (resp. order prime to $p$ ).

The Manin-Mumford conjecture states that, for an irreducible closed subvariety $X$ of $A$, if $\operatorname{Tor}(A) \cap X$ is Zariski dense in $X$, then $X$ is a translate of an abelian subvariety of $A$. For a prime number $p$, we can also consider the " $p$-primary version" (resp. "prime-to- $p$ version") by considering $\operatorname{Tor}_{p}(A)$ (resp. $\left.\operatorname{Tor}^{p}(A)\right)$ instead of $\operatorname{Tor}(A)$. This conjecture was originally proposed by Lang for curves embedded in their Jacobian varieties ([L1]). A first partial result was obtained by Bogomolov, who proved the " $p$-primary version" for each $p$ using $p$-adic Hodge theory ( $\overline{\mathrm{Bog}} \mid)$. The first full proof was obtained by Raynaud by rigid analytic methods (Ra1], Ra2 ). Up to now, several different proofs and generalizations are known by many people such as Hindry, Hrushovski, Ullmo, Szpiro, Zhang, Pink, Roessler ([Hi], $\mathrm{Hr}$,

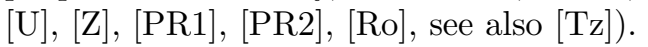

The aim of this paper is to give a short proof of the "prime-to- $p$ version" of the Manin-Mumford conjecture for an abelian variety over a number field, when it has supersingular reduction at a prime dividing $p$, by combining the methods of Bogomolov, Hrushovski, and Pink-Roessler (see Remark 3 for a difference between the proof here and previously known ones).

Recall that an abelian variety $A$ over a field $k$ of characteristic $p>0$ is supersingular if and only if $A \otimes_{k} \bar{k}$ is isogenous to a power of an elliptic curve $E$ over $\bar{k}$ such

Received by the editors December 7, 2004 and, in revised form, April 28, 2005.

2000 Mathematics Subject Classification. Primary 14K12; Secondary 11G10, 14G15.

(C)2006 American Mathematical Society Reverts to public domain 28 years from publication 2857 
that $E(\bar{k})$ has no nontrivial $p$-torsion point. There are many other characterizations (e.g. [LO]).

Theorem 1. Let $A$ be an abelian variety of dimension $g$ over a number field $K$. Assume that $A$ has supersingular reduction at a prime $v$ dividing $p$. Let $F$ be the residue field at $v$. Let $X$ be an irreducible closed subvariety of $A$ defined over $K$. If $\operatorname{Tor}^{p}(A) \cap X$ is Zariski dense in $X$, then $X$ is a translate of an abelian subvariety of $A$, that is, $X=A^{\prime}+a$, where $A^{\prime}$ is an abelian subvariety of $A$ and $a \in A$.

Proof. Let $A_{F}$ be the reduction of $A$ at $v$, which is a supersingular abelian variety over $F$. Let $q$ be the cardinality of $F$, which is a power of $p$. Let $\sigma \in$ $\operatorname{Gal}(\bar{F} / F)$ be the $q$-th power Frobenius automorphism of $\bar{F}$. For a prime number $\ell \neq p$, the characteristic polynomial of $\sigma$ acting on the $\ell$-adic Tate module $V_{\ell} A_{F}:=\left(\varliminf_{n} A_{F}\left[\ell^{n}\right]\right) \otimes_{\mathbb{Z}_{\ell}} \mathbb{Q}_{\ell}$ has coefficients in $\mathbb{Z}$ and is independent of $\ell \neq p$. Let $\alpha_{1}, \ldots, \alpha_{2 g}$ be the eigenvalues $\sigma$ acting on $V_{\ell} A_{F}$. It is known that, for each $i$ and each embedding $\iota: \mathbb{Q}\left(\alpha_{i}\right) \hookrightarrow \mathbb{C}$, the complex absolute value of $\iota\left(\alpha_{i}\right)$ is $q^{1 / 2}$ (e.g. [Mi], $\mathrm{Mu}$ ).

Since $A_{F}$ is supersingular, we know that $\alpha_{i}^{2} / q$ are algebraic numbers whose absolute values are 1 at all finite and infinite primes. Hence $\alpha_{i}^{2} / q$ are roots of unity (e.g. [L2, Chapter V, $\S 1]$ ). Therefore, there is an integer $m \geq 1$ such that $\alpha_{1}^{2 m}=\cdots=\alpha_{2 g}^{2 m}=q^{m}$. Since $\sigma$ acts semisimply on $V_{\ell} A_{F}$ ([Ta]), this means that $\sigma^{2 m} \cdot x=\left[q^{m}\right](x)$ for all $x \in \operatorname{Tor}^{p}\left(A_{F}\right)$. Take a lift $\widetilde{\sigma} \in \operatorname{Gal}(\bar{K} / K)$ of $\sigma$. Since $\operatorname{Tor}^{p}(A)$ is naturally isomorphic to $\operatorname{Tor}^{p}\left(A_{F}\right)$, we have $\tilde{\sigma}^{2 m} \cdot x=\left[q^{m}\right](x)$ for all $x \in \operatorname{Tor}^{p}(A)$. On the other hand, since $X$ is defined over $K$, the set $X(\bar{K})$ is stable by the action of $\operatorname{Gal}(\bar{K} / K)$. Hence we have $\left[q^{m}\right](x) \in X$ for all $x \in \operatorname{Tor}^{p}(A) \cap X$. Since $\operatorname{Tor}^{p}(A) \cap X$ is Zariski dense in $X$, we conclude that $\left[q^{m}\right](X)=X$.

Therefore, $X$ is a translate of an abelian subvariety of $A$ by the same argument as in Bog, Théorème 3]. We reproduce the proof here for the completeness of the paper. After replacing $A$ by its quotient by $\operatorname{Stab}_{A}(X):=\{a \in A \mid X+a=X\}$, we may assume $\operatorname{Stab}_{A}(X)$ is trivial. We shall prove $X$ is a point. Let $d$ be the dimension of $X$, and put $n:=q^{m}$. Consider the action of $[n]$ on the $\ell$-adic cycle class $[X] \in H^{2(g-d)}\left(A \otimes_{K} \bar{K}, \mathbb{Q}_{\ell}(g-d)\right)$ for some $\ell$. Since $[n]: A \rightarrow A$ is an étale Galois covering of $A$ and the Galois group is $A[n]$ acting on $A$ by translation, we have $[n]^{-1}(X)=\bigcup_{a \in A[n]}(X+a)$. Since $\operatorname{Stab}_{A}(X)$ is trivial, we know that $X+a$ are different for all $a \in A[n]$. Hence we have $[n]^{*}[X]=\sum_{a \in A[n]}[X+a]=n^{2 g}[X]$. Since $[n]^{*}$ acts on $H^{2(g-d)}\left(A \otimes_{K} \bar{K}, \mathbb{Q}_{\ell}(g-d)\right)$ via multiplication by $n^{2(g-d)}$, we have $n^{2(g-d)}=n^{2 g}$. Therefore, we have $d=0$ and $X$ is a point.

Remark 2. By a theorem of Elkies, if $A$ is isogenous to a power of an elliptic curve and $K$ admits a real embedding, there are infinitely many primes of supersingular reduction (E1], E2]). However, no general result seems known up to now for a general abelian variety over a number field.

Remark 3. Here we compare the proof above and the proofs previously obtained by Bogomolov, Hrushovski, and Pink-Roessler. Bogomolov proved, for each $p$, that there exists an element $\sigma \in \operatorname{Gal}(\bar{K} / K)$ which acts on the $p$-adic Tate module via multiplication by an integer $n>1$ ( Bog. Corollaire 2]). The proof of this fact relies on a study of $p$-adic Galois representations which have Hodge-Tate decomposition at a prime dividing $p$. It seems very difficult to determine $\sigma$ and $n$ explicitly. On the other hand, Hrushovski considered a lift of the Frobenius element at a 
prime of good reduction. However, the Frobenius element does not act on the Tate module via homothety in general. Hrushovski overcame this difficulty by using the model theory of difference fields $([\mathrm{Hr}])$. Pink-Roessler, inspired by Hrushovski's work, obtained a new proof using only classical algebraic geometry ([PR1], see also PR2]). Their proof is very short and illuminating, but we need a trick for replacing $A$ by a power of it (see the proof of [PR1, Theorem 1.2]). The observation of this paper is that a power of a lift of the Frobenius element at a supersingular prime acts on the prime-to- $p$ torsion points via nontrivial homothety.

Remark 4. It is true but not automatic that a combination of the "p-primary version" and the "prime-to- $p$ version" implies the full Manin-Mumford conjecture. We need some Galois theoretic results for proving such an implication (see Ra2, $\S 8.1]$, see also [Ro]). It seems difficult to obtain the full Manin-Mumford conjecture by simply generalizing the method of this paper because it seems difficult to control the action of the Galois group on $\operatorname{Tor}_{p}(A)$. Note that, in [PR1, §3], to cover all torsion points, Pink-Roessler took lifts of the Frobenius elements at two primes of good reduction of different characteristics, and used a deep ramification-theoretic result of Serre. In Ro, Roessler used a result of Boxall instead of Serre's result. Finally, we note that the idea of finding an element in the Galois group acting on the Tate module via nontrivial homothety to prove the Manin-Mumford conjecture is classical and due to Lang ([L1, §3], see also [Tz, Conjecture 5.1]).

\section{ACKNOWLEDGMENTS}

The author would like to thank Seidai Yasuda and Teruyoshi Yoshida for valuable comments and discussions. He would also like to thank Florian Breuer and Damien Roessler for comments and remarks. After the first version of this paper was written, Sinnou David informed the author that an idea of using a prime of supersingular reduction already appeared in BZ] in a different context. This paper was written during the author's stay at Harvard in October and November of 2004. He would like to thank the Department of Mathematics of Harvard University for its cordial hospitality. The author was supported by the Japan Society for the Promotion of Science Research Fellowships for Young Scientists.

\section{REFERENCES}

[Bog] F. A. Bogomolov, Sur l'algébricité des représentations l-adiques, C. R. Acad. Sci. Paris Sér. A-B 290 (1980), no. 15, A701-A703. MR0574307 (81c:14025)

[BZ] E. Bombieri, U. Zannier, Heights of algebraic points on subvarieties of abelian varieties, Ann. Scuola Norm. Sup. Pisa Cl. Sci. (4) 23 (1996), no. 4, 779-792. MR1469574|(98j:11043)

[E1] N. D. Elkies, The existence of infinitely many supersingular primes for every elliptic curve over Q, Invent. Math. 89 (1987), no. 3, 561-567. MR0903384 (88i:11034)

[E2] N. D. Elkies, Supersingular primes for elliptic curves over real number fields, Compositio Math. 72 (1989), no. 2, 165-172. MR 1030140(90i:11058)

[Hi] M. Hindry, Autour d'une conjecture de Serge Lang, Invent. Math. 94 (1988), no. 3, 575-603. MR0969244 (89k:11046)

[Hr] E. Hrushovski, The Manin-Mumford conjecture and the model theory of difference fields, Ann. Pure Appl. Logic 112 (2001), no. 1, 43-115. MR.1854232 (2003d:03061)

[L1] S. Lang, Division points on curves, Ann. Mat. Pura Appl. (4) 70 (1965), 229-234. MR0190146 (32:7560)

[L2] S. Lang, Algebraic number theory, Addison-Wesley Publishing Co., Inc., Reading, Mass., 1970. MR0282947 (44:181)

[Mi] J. S. Milne, Abelian varieties, in Arithmetic geometry (Storrs, Conn., 1984), 103-150, Springer, New York, 1986. MR0861974 
[Mu D. Mumford, Abelian varieties, Published for the Tata Institute of Fundamental Research, Bombay, 1970. MR0282985 (44:219)

[LO] K.-Z. Li, F. Oort, Moduli of supersingular abelian varieties, Lecture Notes in Math., 1680, Springer, Berlin, 1998. MR 1611305 (99e:14052)

[PR1] R. Pink, D. Roessler, On Hrushovski's proof of the Manin-Mumford conjecture, in Proceedings of the International Congress of Mathematicians, Vol. I (Beijing, 2002), 539-546, Higher Ed. Press, Beijing, 2002. MR1989204 (2004f:14062)

[PR2] R. Pink, D. Roessler, On $\psi$-invariant subvarieties of semiabelian varieties and the Manin-Mumford conjecture, J. Algebraic Geom. 13 (2004), no. 4, 771-798. MR2073195 (2005d:14061)

[Ra1] M. Raynaud, Courbes sur une variété abélienne et points de torsion, Invent. Math. 71 (1983), no. 1, 207-233. MR0688265 (84c:14021)

[Ra2] M. Raynaud, Sous-variétés d'une variété abélienne et points de torsion, in Arithmetic and geometry, Vol. I, 327-352, Progr. Math., 35, Birkhäuser, Boston, Boston, MA, 1983. MR0717600 (85k:14022)

[Ro] D. Roessler, A note on the Manin-Mumford conjecture, preprint, math.NT/0409083, 2004.

[Ta] J. Tate, Endomorphisms of abelian varieties over finite fields, Invent. Math. 2 (1966), 134-144. MR0206004 (34:5829)

[Tz] P. Tzermias, The Manin-Mumford conjecture: a brief survey, Bull. London Math. Soc. 32 (2000), no. 6, 641-652. MR 1781574 (2001g:11091)

[U] E. Ullmo, Positivité et discrétion des points algébriques des courbes, Ann. of Math. (2) 147 (1998), no. 1, 167-179. MR1609514 (99e:14031)

[Z] S.-W. Zhang, Equidistribution of small points on abelian varieties, Ann. of Math. (2) 147 (1998), no. 1, 159-165. MR1609518 (99e:14032)

Department of Mathematics, Faculty of Science, Kyoto University, Kyoto 606-8502, JAPAN

E-mail address: tetsushi@math.kyoto-u.ac.jp 